\title{
Self-perceived Mental Health Status and Uptake of Fecal Occult Blood Test for Colorectal Cancer Screening in Canada: A Cross-Sectional Study
}

\author{
Celestin Hategekimana ${ }^{1}$, Mohammad Karamouzian ${ }^{1,2^{*}}$
}

\begin{abstract}
Background: While colorectal cancer (CRC) is one of the most preventable causes of cancer mortality, it is one of the leading causes of cancer death in Canada where CRC screening uptake is suboptimal. Given the increased rate of mortality and morbidity among mental health patients, their condition could be a potential barrier to CRC screening due to greater difficulties in adhering to behaviours related to long-term health goals. Using a population-based study among Canadians, we hypothesize that self-perceived mental health (SPMH) status and fecal occult blood test (FOBT) uptake for the screening of CRC are associated.

Methods: The current study is cross-sectional and utilised data from the Canadian Community Health Survey 2011-2012. Multinomial logistic regression analysis was undertaken to assess whether SPMH is independently associated with FOBT uptake among a representative sample of 11386 respondents aged 50-74 years.

Results: Nearly half of the respondents reported having ever had FOBT for CRC screening, including 37.28\% who have been screened within two years of the survey and $12.41 \%$ who had been screened more than two years preceding the survey. Respondents who reported excellent mental health were more likely to have ever been screened two years or more before the survey (adjusted odds ratio $[\mathrm{AOR}]=2.08 ; 95 \% \mathrm{CI}, 1.00-4.43$ ) and to have been screened in the last two years preceding the survey $(\mathrm{AOR}=1.53 ; 95 \% \mathrm{CI}, 0.86-2.71$ ) than those reported poor mental health status.

Conclusion: This study supports the association between SPMH status and FOBT uptake for CRC screening. While the efforts to maximize CRC screening uptake should be deployed to all eligible people, those with poor mental health may need more attention.

Keywords: Screening, Colorectal Cancer (CRC), Fecal Occult Blood Test (FOBT), Self-perceived Mental Health (SPMH) Status

Copyright: $\odot 2016$ by Kerman University of Medical Sciences

Citation: Hategekimana C, Karamouzian M. Self-perceived mental health status and uptake of fecal occult blood test for colorectal cancer screening in Canada: a cross-sectional study. Int J Health Policy Manag. 2016;5(6):365-371. doi:10.15171/ijhpm.2016.14
\end{abstract}

\section{Article History:}

Received: 13 September 2015 Accepted: 6 February 2016 ePublished: 12 February 2016

\section{*Correspondence to:}

Mohammad Karamouzian

Email: Karamouzian.m@alumni.ubc.ca

\section{Key Messages}

Implications for policy makers

- Regular fecal occult blood testing for colorectal cancer (CRC) screening among all eligible Canadian population is suboptimal. Given the efforts made since 2011-2012 and increasing rates of screening, it would be interesting to see how deploying efforts/strategies plays out in the next CRC screening uptake of Canadians.

- Individuals with poor self-perceived mental health (SPMH) status may underutilize CRC screening programs. Canadian policy-makers may need to work with screening programs to further emphasize the importance of CRC screening with an eye for those with mental health conditions.

Implications for public

Colorectal cancer (CRC) is one of the most preventable causes of death from cancer. Prevention and early detection through screening could significantly reduce CRC mortality. In high-income countries like Canada where CRC screening programs are available and mostly covered by universal health insurance, efforts to increase the screening uptake cannot be overemphasized. People aged 50 to 74 years, to whom the screening is recommended, should understand that one of the unique opportunities to prevent death from CRC is to participate regularly in the recommended screening programs, in particular, those with poor mental health conditions.

\section{Background}

Despite being one of the most preventable causes of cancer, colorectal cancer (CRC) is one of the leading causes of cancer mortality globally. ${ }^{1}$ In Canada, CRC is the third most commonly diagnosed cancer and the second and the third leading cause cancer-related death in men and women, respectively. ${ }^{2,3}$ In 2015, the Canadian Cancer Society estimated 25100 Canadians to be diagnosed with CRC and 9300 Canadians to die from the disease; accounting for $12 \%$ of all cancer-related deaths. ${ }^{3}$ CRC screening using a fecal occult blood test (FOBT) as a preventive intervention for early detection of CRC, reduces the disease burden with up to a 
90\% cure rate in the case of early detection. ${ }^{1,4}$ While biennial CRC screening with FOBT is recommended in Canada for people aged 50 to 74 years, a survey of CRC screening in 2011 showed that only half of these people had been screened with FOBT, and participation rates varied across provinces of Canada. ${ }^{5,6}$

Previous studies have suggested that socio-demographic factors (eg, age, marital status, education, income, and race), family history (eg, family or personal history of cancer and chronic diseases), healthcare utilization (eg, health insurance coverage, regular contact with a physician and flu shot), awareness factors (eg, knowledge of CRC, attitude towards CRC and screening, perceived risk of developing CRC), past screening behavior (eg, mammography, pap smear, prostate cancer screening), and lifestyle (eg, history of smoking, alcohol intake, and physical activity) affect screening behaviors. ${ }^{7-15}$ Other factors like mental health disorders may also influence CRC screening participation. ${ }^{16,17}$ Mental health patients have an increased rate of morbidity and mortality. ${ }^{18}$ For example, mental health conditions have been associated with lower mammography rates, ${ }^{18-20}$ pap smears, ${ }^{21,22}$ and cholesterol testing. ${ }^{22}$ This could suggest mental health conditions as a potential barrier to CRC screening due to greater difficulties in adhering to behaviours related to long-term health goals or larger emphasis put on the management of the mental health condition compared to seeking preventive healthcare. ${ }^{23,24}$ Nonetheless, the evidence on the association of CRC and FOBT uptake is equivocal in the literature. A study by Calderwood and colleagues analysing data from the 2009 Medical Expenditures Panel Survey in the United States suggested that individuals with depression were more likely to have been screened for CRC, ${ }^{16}$ while Kodl and colleagues looked at veterans from Minneapolis and found that veterans who were diagnosed with mental health disorders were least likely to participate in the screening programs for CRC. ${ }^{17}$ Given the low CRC screening participation rate $^{6}$ and the high burden of mental health conditions in Canada (20\% of Canadians experiencing mental illness during their life course $)^{25}$ as well as mixed evidence about the association of mental health and CRC screening behaviour, ${ }^{16,17}$ we aimed to examine the association of self-perceived mental health status (SPMH) and FOBT uptake for CRC screening using a population-based, national health survey of Canadians. Understanding the relationship between mental health and subsequent preventive health seeking behaviors such as CRC screening, offers the potential to have a population-health impact in Canada, especially for individuals who may already be in contact with the healthcare system for mental health concerns. Identification of this association could also inform policy-makers in developing targeted interventions to help meet the needs of this sub-population and prioritize funding resources more efficiently.

\section{Methods \\ Study Design}

The Canadian Community Health Survey (CCHS) is a biennial, population-based cross-sectional survey carried out by the Statistics Canada using complex multi-stage sampling to produce representative data on health measures, health behaviours and health services utilization among Canadian population. ${ }^{26}$ In the CCHS 2011-2012 cycle, 124929 participants were interviewed across Canada. People aged at least 12 years were included in the survey yielding a representative sample of around $98 \%$ of the Canadian population. ${ }^{26}$ Those not represented (2\%) include people who live on Indian Reserves or Crown Lands, institutional residents, and certain remote regions of Canada as well as full-time Canadian Forces. ${ }^{26}$ Further details on CCHS and methodology are explained by Statistics Canada. ${ }^{26}$

Analytic Sample

This analysis of the association between SPMH and FOBT uptake included people aged 50-74 years with valid responses to SPMH, FOBT, and potential confounding variables of age,

\begin{tabular}{|c|}
\hline $\begin{array}{l}\text { CCHS 2011-2012 cycle respor } \\
\qquad \mathbf{N}=124929\end{array}$ \\
\hline $\begin{array}{l}\text { Respondents who were asked if they e } \\
\qquad n=42367\end{array}$ \\
\hline $\begin{array}{l}\text { Respondents aged } 50 \text { to } 74 \\
\qquad \mathbf{n}=\mathbf{2 3} 4 \mathbf{4 7 6}\end{array}$ \\
\hline $\begin{array}{l}\text { Respondents who provided valid responses to main ex } \\
\qquad \mathbf{n}=\mathbf{2 3} 329\end{array}$ \\
\hline $\begin{array}{l}\text { Respondents who provided valid responses to the } p c \\
\qquad \mathbf{n = 2 2 2 4 0}\end{array}$ \\
\hline $\begin{array}{l}\text { Subtract number of respondents who had FOBT for treatment/mec } \\
\qquad n=19915\end{array}$ \\
\hline $\begin{array}{l}\text { Exclude respondents who had colonosco } \\
\qquad n=11386\end{array}$ \\
\hline $\begin{array}{l}\text { Final analytic sample } \\
\qquad n=11386\end{array}$ \\
\hline
\end{tabular}

CCHS, Canadian Community Health Survey; FOBT, Fecal Occult Blood Test

Figure. Selection of Study Sample From CCHS Respondents. 
gender, immigration status, education attainment, and race in this association (Figure). Furthermore, only respondents who had FOBT for the following reasons were included in the analysis: family history of CRC, part of regular checkup/routine screening, and age. Respondents with invalid responses (Do not Know, Refusal, Not Stated) to any of the study variables were not considered in the current analysis, as were respondents who had the FOBT for medical follow-up of a problem or other unspecified reasons. Respondents who had colonoscopy/sigmoidoscopy were also excluded from the current analysis.

Of the 124929 respondents to the CCHS 2011-2012 cycle, $33.91 \%$ were 35 years old and over and were asked if they had ever had an "FOBT test" $(\mathrm{n}=42367)$. Of these respondents, $55.41 \%$ were aged 50 to 74 years old $(n=23476)$; of whom, $23329(99.45 \%)$ provided valid responses to SPMH status and FOBT $(\mathrm{n}=23329)$. Of these, $4.66 \%$ were excluded from the analysis for invalid responses to potential confounding variables of education $(n=584)$, immigration status $(n=196)$, and race $(n=309)$, and another $10.45 \%$ were excluded from the analysis because they have had FOBT testing for followup of a problem $(n=1816)$ or follow-up of a treatment $(n=82)$ or other non-specified reasons $(n=427)$. In addition, individuals who had colonoscopy/sigmoidoscopy $(n=8529)$ were excluded. Therefore, the final analytic sample included 11386 respondents (Figure).

\section{Study Variables}

The outcome variable in the current analysis was FOBT uptake for CRC screening (a three-level variable) as per current recommendations in Canada. The Canadian Cancer Society suggests men and women aged $\geq 50$ to have a screening test, at least, every two years. ${ }^{5}$ The survey respondents with at least 35 years of age were asked "Have you ever had FOBT test?" followed by "When was the last time FOBT test done?" For the purpose of this study, however, only respondents aged 50 to 74 years were considered for the analysis as the incidence of CRC increases with age and CRC risk is highest for individuals aged $\geq 50 .{ }^{27}$ Respondents who answered "No" to the first FOBT survey question were categorized as "No FOBT" while those who answered "Yes" were asked a subsequent question and then categorized into "FOBT within two years" if they had an FOBT within the two years preceding the survey, and "FOBT $\geq 2$ years" if they have had an FOBT within more than two years of the survey.

The explanatory variable in this study was SPMH which was obtained using the following question: "In general, would you say your mental health is excellent, very good, good, fair or poor?" Previous research has indicated that SPMH status is a "general indication of the number of people in the population suffering from some form of mental disorder, mental or emotional problems or distress, not necessarily reflected in self-perceived health," and is associated with multiitems mental health scales such as K6 scale of psychological distress, mental health subscales of the SF-12 health status survey, and patient health questionnaire (PHQ-2) depression screener. ${ }^{28}$ Self-reported health has also been shown to be highly correlated with overall health outcomes and can be considered to provide a valid measurement of mental health disorders. ${ }^{29}$
Potential confounders included in the current analysis were age (5-year age groupings), gender (males vs. females), level of education completed (less than secondary education, secondary education, completion or vs. some/completed post-secondary education), race (White vs. non-White), and immigration status (yes vs. no).

\section{Statistical Analysis}

All statistical analyses were undertaken using SAS statistical software version 9.3. The CCHS multistage cluster sampling design was accounted for in the analyses using survey procedures in SAS to apply survey weights developed by Statistics Canada. ${ }^{30}$

Descriptive statistics were calculated for the analytic sample. Bivariable multinomial logistic regression analyses were conducted to investigate the association between SPMH and FOBT uptake, and between each of the potential confounders (ie, gender, age, education, race, and immigration status) and FOBT uptake. A multivariable multinomial logistic regression model was constructed to assess the association between SPMH and FOBT uptake while adjusting for the potential confounding effects of gender, age, education, immigration status, and race. The measure of association, odds ratios (OR) (crude and adjusted) with related 95\% CI were reported from the multinomial logistic regression analyses.

\section{Results}

The total study sample $(\mathrm{n}=11386)$ - including 120 who had FOBT for family history of CRC - was unevenly distributed across SPMH with fewer respondents reporting fair $(\mathrm{n}=588$, $5.20 \%)$ and poor $(n=164,1.40 \%)$ mental health status (Table 1 ). Respondents were equally spread across gender and the five age groups, except fewer respondents in the oldest age category (12.70\% aged 70-74 years). Only 1091 (9.58\%) of the respondents in the study sample identified as non-White, while 6717 (58.99\%) identified as having post-secondary education and 2133 (18.70\%) as being an immigrant (Table 1). Nearly half of the respondents included in the study reported having ever had FOBT for CRC screening, including 4245 (37.28\%) who had FOBT within two years of the survey and $1413(12.41 \%)$ who had FOBT $\geq 2$ years preceding the survey. The FOBT uptake rate varied by SPMH; the better the reported mental health status, the more the screening by the respondents (Table 1). The uptake rate also varied by the potential confounding variables with a higher number of male and younger respondents (50-54 years) reporting having never had FOBT $53.30 \%$ and $66.80 \%$, respectively.

In the unadjusted multinomial logistic regression model, higher odds of FOBT uptake was related to better SPMH for both FOBT uptake within two years and $\geq 2$ years of the survey versus never had FOBT. The association between SPMH and FOBT uptake $\geq 2$ years of the survey exhibited the strongest and statistically significant association (Table 2). Compared to poor SPMH, the odds of ever had FOBT for CRC screening increased from 1.41 (95\% CI: $0.58,3.40)$ among those who reported fair mental health to 2.39 (95\% CI: 1.15, 4.95) for those who reported very good mental health, and 2.18 (95\% CI: $1.04,4.57)$ for those who reported excellent mental health (Table 2). In the multivariable multinomial logistic regression model adjusted for gender, age, race, immigration status, and 
Table 1. Characteristics of CCHS Sample (2011-2012) Cycle, Investigation of the Association of SPMH Status and Uptake of FOBT

\begin{tabular}{|c|c|c|c|c|c|}
\hline & \multicolumn{2}{|c|}{ Overall Study Sample $^{a}$} & \multicolumn{3}{|c|}{ Study Sample by FOBT Uptake } \\
\hline & $n=11386$ & Percent & Had FOBT Within 2 Years (\%) & Had FOBT $\geq 2$ Years (\%) & Never Had FOBT (\%) \\
\hline \multicolumn{6}{|l|}{ Reported mental health } \\
\hline Excellent & 3981 & 35.00 & 39.20 & 11.70 & 49.10 \\
\hline Very good & 4100 & 36.01 & 38.30 & 13.40 & 48.20 \\
\hline Good & 2553 & 22.40 & 34.20 & 12.60 & 53.20 \\
\hline Fair & 588 & 5.20 & 32.10 & 10.20 & 57.70 \\
\hline \multicolumn{6}{|l|}{ Gender } \\
\hline Male & 5117 & 44.90 & 34.90 & 11.80 & 53.30 \\
\hline Female & 6269 & 55.10 & 39.20 & 12.90 & 47.90 \\
\hline \multicolumn{6}{|l|}{ Immigrant } \\
\hline Yes & 2133 & 18.70 & 38.80 & 12.40 & 48.80 \\
\hline No & 9253 & 81.20 & 36.90 & 12.40 & 50.70 \\
\hline \multicolumn{6}{|l|}{ Age group } \\
\hline $55-59$ years & 2714 & 23.80 & 35.20 & 12.90 & 51.80 \\
\hline $60-64$ years & 2550 & 22.40 & 41.50 & 14.10 & 44.40 \\
\hline $65-69$ years & 2077 & 18.30 & 43.80 & 14.30 & 41.90 \\
\hline $70-74$ years & 1448 & 12.70 & 43.70 & 16.00 & 40.30 \\
\hline \multicolumn{6}{|l|}{ Race } \\
\hline White & 10295 & 90.42 & 37.75 & 12.71 & 49.55 \\
\hline Non-White & 1091 & 9.58 & 32.91 & 9.62 & 57.47 \\
\hline \multicolumn{6}{|l|}{ Education } \\
\hline Less than secondary & 2202 & 19.34 & 32.56 & 13.40 & 54.04 \\
\hline Secondary & 2467 & 21.67 & 36.16 & 12.32 & 51.52 \\
\hline Post-secondary & 6717 & 58.99 & 39.24 & 12.12 & 48.64 \\
\hline
\end{tabular}

Abbreviations: FOBT, fecal occult blood test; SPMH, self-perceived mental health; CCHS, Canadian Community Health Survey.

All percentages are rounded.

adjusted for age, gender, race, immigration status, and education.

education, the odds of FOBT uptake were attenuated, but similar patterns were observed with stronger associations for ever had FOBT with increasing levels of SPMH. The individuals who perceived better mental health status were more likely to have been screened (referring to FOBT uptake in our study) than those who perceived poor mental health status (Table 2). However, in both unadjusted and adjusted multinomial logistic models, most of the $95 \%$ CIs were wide and included " 1 " making estimates less plausible, except for the CIs around the estimates for ever had FOBT associated with the higher levels of mental health (good, very good, and excellent).

With respect to the confounding variables, female respondents were more likely to have ever been screened and to have been screened in the last two years than male respondents. The odds of ever had FOBT and up to date FOBT increased with age. The variables with the strongest confounding effects were age and education, with each variable attenuating the main effect by more than $10 \%$ each in adjusted models. Since individuals may get a colonoscopy or sigmoidoscopy instead of FOBT for CRC screening, a sensitivity analysis was performed by further investigation of the association between SPMH and both regular and irregular testing for colonoscopy or sigmoidoscopy, which found no significant association. Lastly, our study had enough power $(>0.99)$ to detect a difference in effect size between normal SPMH (excellent, very good, good) and poor SPMH in the two categories: up to date FOBT compared to no FOBT as well as ever had FOBT compared to no FOBT at an alpha of .05.

\section{Discussion}

The current study used a national representative survey of 11386 individuals to evaluate the association of SPMH status with FOBT uptake for screening of CRC among Canadians aged 50 to 74 years. The study contributes to the new understanding of the association between SPMH and FOBT uptake for CRC screening, demonstrating that the better the reported mental health, the higher the odds of CRC screening. However, our findings do not show any statistically significant difference between individuals who had FOBT within two years preceding the survey and those who had never had the FOBT. While public health interventions to increase CRC screening uptake should target all people, those with poor mental health status may need particular attention, as our results suggest that they were not as likely to have ever had a FOBT for screening of the CRC.

Consistent with previously published studies, mental health status is not associated with up to date FOBT uptake. Heflin and colleagues did not suggest any relationship between FOBT uptake and depression in a survey of elderly people in North Carolina. ${ }^{31}$ Likewise in their study at one veterans administration center, Yee et al demonstrated that veterans diagnosed with mental health conditions were as likely to participate in CRC screening programs as those without mental health disorders. ${ }^{32}$ However, their study was underpowered with a non-representative sample. Previous studies have not explored the association between perceived mental health and irregular FOBT uptake and dichotomized CRC screening test uptake with irregular uptake combined 
Table 2. Unadjusted and Adjusted Multinomial Logistic Regression Results for Association of SPMH Status and Uptake to FOBT, CCHS Sample (20112012)

\begin{tabular}{|c|c|c|c|c|}
\hline & \multicolumn{2}{|c|}{ Unadjusted } & \multicolumn{2}{|c|}{ Adjusted $^{\mathrm{a}}$} \\
\hline & $\begin{array}{c}\text { Had FOBT Within } 2 \text { Years } \\
\text { vs. } \\
\text { Never Had FOBT } \\
\text { OR (95\% Cls) }\end{array}$ & $\begin{array}{c}\text { Had FOBT } \geq 2 \text { years } \\
\text { vs. } \\
\text { Never Had FOBT } \\
\text { OR ( } 95 \% \text { Cls })\end{array}$ & $\begin{array}{c}\text { Had FOBT Within } 2 \text { Years } \\
\text { vs. } \\
\text { Never Had FOBT } \\
\text { OR ( } 95 \% \mathrm{Cls})\end{array}$ & $\begin{array}{c}\text { Had FOBT } \geq 2 \text { Years } \\
\text { vs. } \\
\text { Never Had FOBT } \\
\text { OR ( } 95 \% \text { Cls) }\end{array}$ \\
\hline \multicolumn{5}{|l|}{ Reported mental health } \\
\hline Poor & Reference & Reference & Reference & Reference \\
\hline Fair & $1.61(0.79,3.30)$ & $1.41(0.58,3.40)$ & $1.61(0.81,3.19)$ & $1.42(0.59,3.41)$ \\
\hline Very good & $1.66(0.94,2.94)$ & $2.39(1.15,4.95)$ & $1.53(0.86,2.72)$ & $2.32(1.02,4.88)$ \\
\hline Excellent & $1.73(0.98,3.03)$ & $2.18(1.04,4.57)$ & $1.53(0.86,2.71)$ & $2.08(1.00,4.43)$ \\
\hline \multicolumn{5}{|l|}{ Gender } \\
\hline Male & Reference & Reference & Reference & Reference \\
\hline Female & $1.33(1.12,1.58)$ & $1.25(0.96,1.62)$ & $1.33(1.12,1.57)$ & $1.23(0.94,1.60)$ \\
\hline \multicolumn{5}{|l|}{ Age group } \\
\hline $55-59$ years & $1.83(1.42,2.37)$ & $2.64(1.74,3.98)$ & $1.88(1.46,2.41)$ & $2.67(1.77,4.04)$ \\
\hline $60-64$ years & $2.73(2.11,3.54)$ & $4.26(2.80,6.49)$ & $2.74(2.11,3.56)$ & $4.20(2.75,6.39)$ \\
\hline $65-69$ years & $2.68(2.11,3.41)$ & $4.34(2.78,6.79)$ & $2.81(2.20,3.60)$ & $4.20(2.68,6.57)$ \\
\hline 70-74 years & $2.94(2.27,3.81)$ & $7.36(4.29,12.60)$ & $3.22(2.44,4.23)$ & $7.04(4.06,12.17)$ \\
\hline \multicolumn{5}{|l|}{ Race } \\
\hline Non-White & Reference & Reference & Reference & Reference \\
\hline White & $1.58(1.22,2.04)$ & $2.06(1.23,3.44)$ & $1.56(1.14,2.14)$ & $1.93(1.16,3.23)$ \\
\hline \multicolumn{5}{|l|}{ Education } \\
\hline Less than secondary & Reference & Reference & Reference & Reference \\
\hline Secondary & $1.27(1.00,1.62)$ & $0.70(0.46,1.05)$ & $1.43(1.11,1.83)$ & $0.85(0.58,1.25)$ \\
\hline Post-secondary & $1.37(1.10,1.70)$ & $0.74(0.50,1.08)$ & $1.64(1.32,2.04)$ & $1.00(0.70,1.41)$ \\
\hline \multicolumn{5}{|l|}{ Immigrant } \\
\hline Yes & Reference & Reference & Reference & Reference \\
\hline No & $1.14(0.92,1.41)$ & $1.26(0.91,1.74)$ & $1.02(0.79,1.31)$ & $1.05(0.77,1.44)$ \\
\hline
\end{tabular}

Abbreviations: FOBT, fecal occult blood test; SPMH, self-perceived mental health; CCHS, Canadian Community Health Survey.

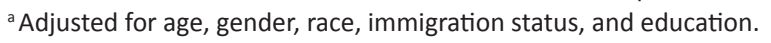

with regular update. ${ }^{17,31,32}$ Dichotomization makes analysis less cumbersome, but may introduce bias in the study result (eg, misclassification). ${ }^{33}$ The current study made use of all information and demonstrated that individuals with lower SPMH (poor or fair) were less likely to have ever had FOBT compared to those with higher perceived mental health (very good or excellent). Looking on up to date screening, however, the difference in FOBT uptake based on one's perceived mental health was not significant.

In contrast, Calderwood and colleagues analysed data from the 2009 Medical Expenditures Panel Survey in the United States and suggested that individuals diagnosed with depression were more likely to participate in CRC screening program, including colonoscopy and FOBT, adjusted for frequency of hospital visits. ${ }^{16}$ Similarly a study by Stecker and colleagues indicated that women diagnosed with depression and aged over 50 years were more likely to uptake colonoscopy compared to those without depression. ${ }^{34}$ The difference between the current study's findings and previous studies may be partially explained by the fact that these studies looked only on diagnosed depression and may have comorbidities leading to increased likelihood of utilizing health services. However, the current study looked on the effect of selfreported mental health that encompassed mental well-being broadly (diagnosed or not). As discussed earlier, SPMH status and multi-items mental health screening scales (eg, K6, SF 12, PHQ-2) are associated but not interchangeable. ${ }^{28}$ In its
CCHS-Mental Health cycle 2012, Statistics Canada collected K6 data which was not associated with FOBT uptake. ${ }^{35}$

While being male was an independent risk factor for CRC, ${ }^{14,36}$ the current study findings suggest that men had slightly lower odds of screening compared to women in both crude and adjusted analyses. This could be to women's higher concerns about health and use of medical services. ${ }^{37}$ Moreover, our results show that the odds of screening for CRC increase with age. This may suggest that as people get older, they are more concerned and cautious about their health and thus, more receptive to public health messages of screening for CRC. Nevertheless, further analysis found that the effect of SPMH status on FOBT uptake was not modified by age.

We would like to acknowledge the limitations of this study. First, reported mental health status can vary from one level to another over time. Given the cross-sectional design of the CCHS, it is impossible, to determine whether the level of current mental health status reported by individuals at the time of the survey is consistent with the level they could have reported, had they been surveyed before undergoing FOBT for CRC screening. Therefore, temporality and causality cannot be established between SPMH and FOBT uptake. However, the current study aimed to evaluate the association between SPMH and FOBT uptake. Second, this study used self-reported data on mental health status and FOBT uptake, and, therefore, this could have introduced bias (eg, nondifferential misclassification) in the study and the potential 
effect of this on the main study findings would be diluting the OR towards the null. Nevertheless, previous researches have suggested self-reported mental health status to be one of the most accurately reported measures with a sensitivity of about $88 \%,{ }^{38}$ while self-reported CRC screening tests (FOBT and/or colonoscopy) highly vary, with low specificity and high sensitivity. ${ }^{39,40}$ Lastly, given the administrative nature of CCHS data, we were not able to include all possible potential confounding variables in our analysis and further explore their association with CRC screening uptake.

In conclusion, the current study findings demonstrate that mental well-being, as measured by self-reported mental health status, is not significantly associated with CRC screening participation. However, it seems that mental health patients may under-participate in CRC screening programs, and, therefore, any endeavor to maximize CRC screening uptake in Canada should take these populations into account.

\section{Acknowledgements}

Authors are grateful to professors Mieke Koehoorn (University of British Columbia, Vancouver, BC, Canada) and Larry Lynd (University of British Columbia, Vancouver, BC, Canada) for their scientific and editorial input over the first draft of the paper.

\section{Ethical issues}

Ethics approval for the use of the CCHS data is covered by the "publicly available data clause (Item 7.10.3)" under the "policy number 89 of the University of British Columbia, Vancouver, BC, Canada: Research and Other Studies Involving Human Subjects" that regulates the utilization of public release data. ${ }^{41}$

Competing interests

Authors declare that they have no competing interests.

Authors' contributions

$\mathrm{CH}$ conceptualized the study and conducted the statistical analysis. Both authors contributed to the interpretation of the findings and drafting the manuscript.

\section{Authors' Affiliations}

'School of Population and Public Health, University of British Columbia, Vancouver, BC, Canada. ${ }^{2}$ Regional Knowledge Hub, and WHO Collaborating Centre for HIV Surveillance, Institute for Futures Studies in Health, Kerman University of Medical Sciences, Kerman, Iran.

\section{References}

1. Hewitson P, Glasziou P, Watson E, Towler B, Irwig L. Cochrane systematic review of colorectal cancer screening using the fecal occult blood test (hemoccult): an update. Am J Gastroenterol. 2008;103(6):1541-1549. doi:10.1111/j.1572-0241.2008.01875.x

2. Canadian Cancer Society's Advisory Committee on Cancer Statistics. Canadian Cancer Statistics; 2015.

3. Canadian Cancer Society. Colorectal cancer statistics 2015. http://www.cancer.ca/en/cancer-information/cancer-type/ colorectal/statistics/?region=bc. Accessed September 12, 2015.

4. Colon Cancer Canada. Fast Facts on Colorectal Cancer 2015. http://coloncancercanada.ca/fast-facts-on-colorectal-cancercrc/. Accessed September 8, 2015.

5. Canadian Cancer Society. Screening for colorectal cancer. http:// www.cancer.ca/en/cancer-information/cancer-type/colorectal/ screening/?region=pe\&sc_prof=1\#ixzz3ELwJ0Xa9. Accessed August 28, 2015. Published 2015.

6. Canadian Partnership Against Cancer. An update on colorectal cancer screening in Canada; 2011.

7. Cole SR, Zajac I, Gregory T, et al. Psychosocial variables associated with colorectal cancer screening in South Australia. Int J Behav Med. 2011;18(4):302-309. doi:10.1007/s12529-0109101-1

8. Dear K, Scott L, Chambers S, Corbett MC, Taupin D. Perception of colorectal cancer risk does not enhance participation in screening. Therap Adv Gastroenterol. 2008;1(3):157-167. doi:10.1177/1756283x08097776

9. Guessous I, Dash C, Lapin P, et al. Colorectal cancer screening barriers and facilitators in older persons. Prev Med. 2010;50(1):310. doi:10.1016/j.ypmed.2009.12.005

10. McCaffery K, Wardle J, Nadel M, Atkin W. Socioeconomic variation in participation in colorectal cancer screening. $J$ Med Screen. 2002;9(3):104-108. doi:10.1136/jms.9.3.104

11. Seeff LC, Nadel MR, Klabunde CN, et al. Patterns and predictors of colorectal cancer test use in the adult US population. Cancer. 2004;100(10):2093-2103. doi:10.1002/cncr.20276

12. Shapiro JA, Seeff LC, Thompson TD, Nadel MR, Klabunde CN, Vernon SW. Colorectal cancer test use from the 2005 National Health Interview Survey. Cancer Epidemiology Biomarkers \& Prevention. 2008;17(7):1623-1630. doi:10.1158/1055-9965.epi07-2838

13. Subramanian S, Klosterman M, Amonkar MM, Hunt TL. Adherence with colorectal cancer screening guidelines: a review. Prev Med. 2004;38(5):536-550. doi:10.1016/j. ypmed.2003.12.011

14. Wardle J, Miles A, Atkin W. Gender differences in utilization of colorectal cancer screening. J Med Screen. 2005;12(1):20-27. doi:10.1258/0969141053279158

15. Zapka JG, Puleo E, Vickers-Lahti M, Luckmann R. Healthcare system factors and colorectal cancer screening. Am J Prev Med. 2002;23(1):28-35. doi:10.1016/s0749-3797(02)00444-0

16. Calderwood AH, Bacic J, Kazis LE, Cabral H. Association between self-reported depression and screening colonoscopy participation. J Ambul Care Manage. 2013;36(4):345. doi:10.1097/jac.0b013e3182a3e73f

17. Kodl MM, Powell AA, Noorbaloochi S, Grill JP, Bangerter AK, Partin MR. Mental health, frequency of healthcare visits, and colorectal cancer screening. Med Care. 2010;48(10):934-939. doi:10.1097/mlr.0b013e3181e57901

18. Colton CW, Manderscheid RW. Congruencies in increased mortality rates, years of potential life lost, and causes of death among public mental health clients in eight states. Prev Chronic Dis. 2006;3(2):A42.

19. Carney CP, Jones LE. The influence of type and severity of mental illness on receipt of screening mammography. J Gen Intern Med. 2006;21(10):1097-1104. doi:10.1111/j.1525-1497.2006.00565.x

20. Egede LE, Grubaugh AL, Ellis C. The effect of major depression on preventive care and quality of life among adults with diabetes. Gen Hosp Psychiatry. 2010;32(6):563-569. doi:10.1016/j. genhosppsych.2010.08.002

21. Leiferman JA, Pheley AM. The effect of mental distress on women's preventive health behaviors. Am J Health Promot. 2006;20(3):196-199.

22. Lord O, Malone D, Mitchell AJ. Receipt of preventive medical care and medical screening for patients with mental illness: a comparative analysis. Gen Hosp Psychiatry. 2010;32(5):519543. doi:10.1016/j.genhosppsych.2010.04.004

23. DiMatteo MR, Lepper HS, Croghan TW. Depression is a risk factor for noncompliance with medical treatment: meta-analysis of the effects of anxiety and depression on patient adherence. Arch Intern Med. 2000;160(14):2101-2107.

24. Katon WJ. Clinical and health services relationships between major depression, depressive symptoms, and general medical illness. Biol Psychiatry. 2003;54(3):216-226.

25. Public Health Agency of Canada. A Report on Mental IIInesses in 
Canada. http://www.phac-aspc.gc.ca/publicat/miic-mmac/sumeng.php. Accessed September 06, 2015. Published 2015.

26. Statistics Canada. Canadian Community Health Survey - Annual Component (CCHS). http://www23.statcan.gc.ca/imdb/p2SV.pl? Function=getSurvey\&SDDS=3226. Accessed March 17, 2015. Published 2015.

27. Division of Cancer Prevention and Control (DCPC). Basic Information About Colorectal Cancer. http://www.cdc.gov/ cancer/colorectal/basic_info/index.htm. Accessed June 20, 2015. Published 2014.

28. Ahmad F, Jhajj AK, Stewart DE, Burghardt M, Bierman AS. Single item measures of self-rated mental health: a scoping review. BMC Health Serv Res.. 2014;14(1):398. doi:10.1186/1472-696314-398

29. Bond J, Dickinson HO, Matthews F, Jagger C, Brayne C, CFAS $M$. Self-rated health status as a predictor of death, functional and cognitive impairment: a longitudinal cohort study. Eur J Ageing. 2006;3(4):193-206. doi:10.1007/s10433-006-0039-8

30. An A, Watts D. New SAS procedures for analysis of sample survey data. SUGI Proceedings. Cary, NC: SAS Institute Inc; 1998.

31. Heflin MT, Oddone EZ, Pieper CF, Burchett BM, Cohen HJ. The effect of comorbid illness on receipt of cancer screening by older people. J Am Geriatr Soc. 2002;50(10):1651-1658. doi:10.1046/ j.1532-5415.2002.50456.x

32. Yee EF, White R, Lee S-J, et al. Mental illness: is there an association with cancer screening among women veterans? Womens Health Issues. 2011;21(4):S195-S202. doi:10.1016/j. whi.2011.04.027

33. Naggara O, Raymond J, Guilbert F, Roy D, Weill A, Altman DG. Analysis by categorizing or dichotomizing continuous variables is inadvisable: an example from the natural history of unruptured aneurysms. Am J Neuroradiol. 2011;32(3):437-440. doi:10.3174/ ajnr.a2425

34. Stecker T, Fortney JC, Prajapati S. How depression influences the receipt of primary care services among women: a propensity score analysis. J Womens Health (Larchmt). 2007;16(2):198205.

35. Statistics Canada. Canadian Community Health Survey - Mental Health (CCHS). http://www23.statcan.gc.ca/imdb/p2SV.pl?Fun ction=getSurvey\&SDDS=5015. Accessed September 8, 2015. Published 2012.

36. Ferlitsch M, Reinhart K, Pramhas $S$, et al. Sex-specific prevalence of adenomas, advanced adenomas, and colorectal cancer in individuals undergoing screening colonoscopy. JAMA. 2011;306(12):1352-1358. doi:10.1001/jama.2011.1362

37. Green CA, Pope CR. Gender, psychosocial factors and the use of medical services: a longitudinal analysis. Soc Sci Med. 1999;48(10):1363-1372. doi:10.1016/S0277-9536(98)00440-7

38. Machlin S, Cohen J, Elixhauser A, Beauregard K, Steiner C. Sensitivity of household reported medical conditions in the medical expenditure panel survey. Med Care. 2009;47(6):618625.

39. Khoja S, McGregor SE, Hilsden RJ. Validation of self-reported history of colorectal cancer screening. Can Fam Physician. 2007;53(7):1192-1197.

40. Partin MR, Grill J, Noorbaloochi S, et al. Validation of selfreported colorectal cancer screening behavior from a mixedmode survey of veterans. Cancer Epidemiology Biomarkers \& Prevention. 2008;17(4):768-776. doi:10.1158/1055-9965.epi-070759

41. The University of British Columbia Board of Governors. Policy No. 89: Research Involving Human Participants; 2002. 